\title{
Oscillating grid generating turbulence near gas-liquid interfaces in shear-thinning dilute polymer solutions
}

\author{
T. Lacassagne ${ }^{*}$ \\ Univ Lyon, INSA de Lyon, Ecole Centrale de Lyon, Université Lyon 1, CNRS, \\ LMFA UMR 5509, 69621 Villeurbanne Cedex, France \\ and Department of Mechanical Engineering, University College London (UCL), \\ London WC1E 7JE, United Kingdom \\ S. Simoëns, ${ }^{\dagger}$ M. EL Hajem, and J.-Y. Champagne \\ Univ Lyon, INSA de Lyon, Ecole Centrale de Lyon, Université Lyon 1, CNRS, LMFA UMR 5509, \\ 69621 Villeurbanne Cedex, France
}

(Received 6 May 2019; accepted 4 March 2020; published 31 March 2020)

\begin{abstract}
Understanding the behavior of liquid phase turbulence near gas-liquid interfaces is of great interest in many fundamental, environmental, or industrial applications. For example, near-surface liquid side turbulence is known to enhance the mass transfers between the two phases. Descriptions of this behavior for air-water systems exist in the literature, but the case of turbulence in a shear-thinning liquid phase below a flat gas-liquid interface has never been considered to the best of our knowledge. This paper consists in an experimental characterization of low Reynolds number, oscillating grid generated, nearsurface turbulence in shear-thinning dilute polymer solutions, in the surface-influenced and in the viscous sublayers. The energy transfer mechanism, known in the water case, is evidenced in dilute polymer solutions. A horizontal damping mechanism, similar to the one introduced by surfactants, is evidenced. The evolution of the viscous sublayer depth can be explained by both viscous and shear-thinning effects, and it appears that a critical polymer concentration may exist within the dilute regime.
\end{abstract}

DOI: 10.1103/PhysRevFluids.5.033301

\section{INTRODUCTION}

Turbulence is known to be a very efficient enhancement mechanism of mixing and mass transfer between phases. In particular, it has been shown that liquid side turbulence enhances air-water mass transfer of low diffusive atmospheric gases at flat interfaces, by locally deforming the liquid side concentration boundary layer [1-3]. These local, near-surface, mixing mechanisms occur in thin sublayers under the interface, typically of $1 \mathrm{~cm}$ to less than $1 \mathrm{~mm}$. To describe them, a precise understanding of the behavior of turbulence near flat gas-liquid interfaces is needed. This question has been open for several decades [4-7] and is arduous to tackle, both numerically and experimentally, because of the small scalar scales involved in the mixing low-diffusivity, high-Schmidt-number species. Studies have so far focused mostly on air-water interfaces, clean (e.g. [5,6]) or polluted $[8,9]$.

Many industrial process yet involve turbulent gas-liquid mass transfer in complex rheology fluids (e.g., bacteria production in fermentation devices [10,11]), and mass transfer models efficient for

\footnotetext{
*tom.lacassagne@gmail.com

${ }_{\dagger}^{\dagger}$ serge.simons@ec-lyon.fr
} 
Newtonian fluids fail to predict mass transfer efficiency in those situations. Accurate estimation of the mass transfer velocity requires a good knowledge of fundamental and local aspects of turbulence at gas liquid interfaces, which depends itself on the rheology of the liquid phase. Understanding near-surface turbulence for complex interface shapes starts by studying the effects of a simplified interface geometry on turbulence properties. Corresponding appropriate experimental studies have never been done in non-Newtonian media to the best of our knowledge.

The aim of this work is to provide an experimental study of near-surface liquid turbulence in a non-Newtonian, shear-thinning fluid, in the few millimeters underneath a flat gas-liquid interface. The rest of the paper is organized into four sections. In the first section the main concepts of nearsurface turbulence in Newtonian fluids are introduced, and the possible effects of non-Newtonian properties on turbulence are evoked. In the second section, the experimental procedure is detailed. Results are given in the third section, and additional discussion and modeling are provided in the last.

\section{BACKGROUND}

In order to describe the interactions between a horizontal free surface and upcoming bulk turbulence, Hunt and Graham [12] and Brumley and Jirka [5] defined two "undisturbed" scales: the horizontal integral length scale and horizontal velocity fluctuations (root mean square value), respectively, $L_{\infty}$ and $u^{\prime}$. These are the values that would be found at the interface location if there were no interface. They thus depend on the bulk turbulence properties only. The depth at which turbulence begins to "see" the interface is called the surface-influenced (or blocking or source) layer and is assumed to be about the size of one integral length scale of the upcoming bulk turbulence $L_{\infty}$ [4,5]. In other words, the surface is supposedly only able to act on eddies once they reach it, and so the maximum depth of influence of this interface is about the size of the largest existing eddies. In the case of a flat horizontal interface, only vertical motion is constrained by the free surface, and so vertical velocity fluctuations in the source layer are supposed to be damped. Energy is transferred from vertical to horizontal motion, and eddies deform and stretch along horizontal dimensions. This pictured view was stated by the theoretical works of Hunt and Graham [4,12], and later confirmed by direct numerical simulations (DNS) $[6,13,14]$ and experiments $[1,2,5]$. Bodart et al. [15] observed that velocity dynamics in the source layer are mostly independent of the surface boundary condition, which suggests that the results of Hunt and Graham's theory applies equally well to clean, contaminated, and no-slip surfaces up to a certain proximity with the interface. This higher level of proximity is defined by another smaller typical depth called the viscous sublayer $\delta_{v}$, which scales as $[5,16]$

$$
\delta_{v}=L_{\infty} \operatorname{Re}_{T}^{-0.5}
$$

where $\operatorname{Re}_{T}$ is the turbulent Reynolds number at the interface, expressed as $\operatorname{Re}_{T}=\frac{\rho u^{\prime} L_{\infty}}{\mu}$, with $\rho$ and $\mu$ the density and dynamic viscosity, respectively. This sublayer is defined as the depth from which an eddy adjusts to the boundary condition set by the interface by viscous effects. Note that Eq. (1) can sometimes be found with a prefactor of 2 [16], and this discrepancy may become important at low Reynolds numbers [Eq. (1) has been verified for $\operatorname{Re}_{T} \geqslant 70$ in Ref. [5], but DNS results exist at lower $\operatorname{Re}_{T}$, as reported in Ref. [16]]. At clean interfaces (i.e., free of surfactant or particles), there is no constraint on the horizontal velocity fluctuations themselves, but on the vertical gradients of the rms of horizontal velocity fluctuations, which fall to zero [6]. According to Hunt [4], this in theory leads only to a reduction in the increase rate (inherited from the bulk turbulence properties) of horizontal velocity fluctuations: the vertical gradients of the rms of horizontal fluctuations are damped in the viscous sublayer. One should stress that for dirty interfaces, the horizontal surface velocity is also constrained. This additional boundary condition then also leads to a decrease in rms of horizontal velocity fluctuations $[2,8,17]$ : the horizontal velocity fluctuations are nil, and their rms' vertical gradient is small at the interface. Several experimental studies $[1,2,5,18]$ and DNS $[6,14,16]$ 
showed that, even if the theory of Hunt [4] holds very well in most of the surface-influenced layer, it does not accurately predict the behavior of horizontal velocity fluctuations in the viscous sublayer.

Moreover, all the previous concepts have not been verified in dilute polymer solutions (DPSs). It is yet known that a very small concentration of polymer diluted in a solvent can drastically change boundary layer interaction, leading to phenomena such as drag reduction [19]. The coil-stretch transformations of polymer chains and their interactions with flow structures are such that polymer molecules can store energy at small scales and give it back to the flow at larger scales [20-23]. This leads to several characteristic features of bulk turbulence in viscoelastic and/or shear-thinning polymer solutions such as a suppression of smallest scales of turbulence [24,25], a large-scale enhancement [24,26,27], and an increased anisotropy [25,28,29].

The goal of this work will thus be to provide information on how turbulence properties are affected by the presence of polymer and of the interface around and within this viscous sublayer.

\section{MATERIALS AND METHODS}

\section{A. Shear-thinning polymer solutions}

Shear-thinning properties are conferred to the liquid by addition of a minute amount of a polymer, xanthan gum (XG), into distilled water. Here XG produced by Kelco under the commercial name Keltrol CG-T is used. Its average molar mass is $M_{w}=3.4 \times 10^{6} \mathrm{~g} \mathrm{~mol}^{-1}$, and its polydispersity equal to 1.12 [30]. XG is chosen for its high resistance to strong shears and extreme temperature and $\mathrm{pH}$ conditions [31]. Such features are useful when using it near a rigid oscillating grid, or if one were to consider extending this work to mass transfer measurements of atmospheric gases. XG yields optically clear non-Newtonian solutions once dissolved in aqueous media. This allows the use of optical methods for liquid phase velocity measurements. This polymer is commonly utilized as a flow additive for non-Newtonian fluid mechanics experiments, but also in the food, process, and oil and gas industries [32]. Its rheology and its properties have been widely studied in the literature [33-35]. Depending on the polymer concentration $C_{\mathrm{XG}}$, one may distinguish between three entanglement regimes: dilute, semidilute, and concentrated [33,34]. In the last two cases, the flow behavior is defined by fluid polymer but most importantly polymer-polymer mechanical or electrical interactions. In this work the focus is made on the dilute regime, for which polymer molecules interact only with the flow and not between each other. It concerns the $C_{\mathrm{XG}}<100 \mathrm{ppm}$ concentration range [34]. The critical transition concentration is notated $C_{D}=100 \mathrm{ppm}$. The shear-thinning behavior of aqueous XG solutions can be modeled by a Carreau-Yasuda (CY) equation

$$
\frac{\mu-\mu_{\infty}}{\mu_{0}-\mu_{\infty}}=\left[1+\left(t_{\mathrm{CY}} \dot{\gamma}\right)^{a}\right]^{\frac{n-1}{a}}
$$

where the values of zero shear rate and infinite shear rate Newtonian plateau (resp. $\mu_{0}$ and $\mu_{\infty}$ ), characteristic timescale $t_{\mathrm{CY}}$, and exponents $a$ and $n$ depend on polymer concentration $C_{\mathrm{XG}}$. Viscosity is measured as a function of the shear rate using a MCR 302 Anton Paar rheometer, and the curves obtained are fitted by a CY model (least-square fitting) to get values of $\mu_{0}, \mu_{\infty}, a, n$, and $t_{\mathrm{CY}}$. The fitting parameters for four concentrations in the dilute regime are reported in Table I.

Viscosity and timescale evolution with polymer concentration are consistent with the ones found by Wyatt and Liberatore [34]. Viscosity values are similar, and timescales measured for the present study are typically one order of magnitude lower than in reference [34] (in which timescales are derived by fitting with a cross model instead of a CY one). It can be due to the fact that the properties of XG are very sensitive to the molar mass $M_{w}$ of the polymer, which depends itself on production and dissolution conditions [31]. In order to avoid the formation of disordered chains during dissolution, and ensure the reproducibility of measurements, special care has to be taken in the process of dissolving XG. Only distilled water is used without adding any salt, and moderate stirring and heating conditions are applied as specified by Garcia-Ochoa et al. [31]. Every solution was used only once and less than $24 \mathrm{hr}$ after preparation. Tests were done before and 
TABLE I. Carreau-Yasuda fitting parameters for the shear-thinning behavior of XG solutions at various concentrations, undisturbed scales, and Reynolds and Deborah numbers.

\begin{tabular}{lccccccccc}
\hline \hline$C_{\mathrm{XG}}[\mathrm{ppm}]$ & $\mu_{0}[\mathrm{mPa} \mathrm{s}]$ & $\mu_{\infty}[\mathrm{mPa} \mathrm{s}]$ & $t_{\mathrm{CY}}[\mathrm{s}]$ & $a$ & $n$ & $u^{\prime}[\mathrm{mm} / \mathrm{s}]$ & $L_{\infty}[\mathrm{mm}]$ & $\mathrm{Re}_{T}$ & $\mathrm{De}$ \\
\hline 0 (water) & 1.00 & 1.00 & 0 & - & - & 4.5 & 24.5 & 110 & 0 \\
10 & 1.30 & 0.99 & 0.08 & 2.00 & 0.60 & 3.0 & 45.6 & 106 & 0.08 \\
25 & 3.97 & 0.95 & 0.45 & 2.00 & 0.57 & 2.0 & 127.8 & 64 & 0.46 \\
50 & 10.94 & 1.09 & 0.55 & 2.00 & 0.50 & 1.5 & 149.1 & 20 & 0.55 \\
100 & 32.78 & 1.05 & 1.58 & 2.00 & 0.50 & 1.0 & 138.4 & 4 & 1.58 \\
\hline \hline
\end{tabular}

after any experimental trial to judge any degradability of the solution. All experiments are done with the certitude that solutions were not degraded. With the procedure used for the fabrication of XG solutions, uncertainty about $C_{\mathrm{XG}}$ lies between about $10 \%$ for lowest polymer concentrations and down to $5 \%$ for higher polymer concentrations. Reproducibility of experiments is checked by repeating experiments at the same concentration with a new solution. The typical variation of root-mean-square velocities between two runs at similar polymer concentration is $\pm 5 \%$.

\section{B. Turbulence generation}

Turbulence is generated in the liquid phase by the mean of an oscillating grid device. It is a common tool in turbulence study since it theoretically creates almost no mean flow and quasihomogeneous and isotropic turbulence in horizontal planes when measured far enough from the grid's top position. The question of mean flow in oscillating grid turbulence (OGT) is yet quite complex: studies have shown that a mean flow always exists for OGT in water $[36,37]$ because of imperfections in the grid/tank alignment and boundary layer effects at the tank's walls. In a separate work [38], we discussed the existing mean flow in OGT and the effects of polymer on it. As for the present study, mean flows in the near-surface region cannot be avoided but are checked to be always at most of the same order of magnitude than turbulence and stationary. With or without mean flow, characterization of turbulence in the tank and at the interface still remains of first and main importance for turbulent mass transfer characterization [3].

The grid setup used here is based on the work of Thompson and Turner [39] and Hopfinger and Toly [40] and sketched in Fig. 1. A cartesian, squared meshed grid with square bars of $7 \mathrm{~mm}$ width is used. The transparent tank has a $277 \mathrm{~mm}$ by $277 \mathrm{~mm}$ inner cross section, the fluid height is set at $H=450 \mathrm{~mm}$, and the distance between the surface and the average grid position is $h=$ $250 \mathrm{~mm}$. In this study only polymer concentration is varied, and all oscillations parameters are kept constant. The frequency is fixed at $1 \mathrm{~Hz}$ and the stroke at $45 \mathrm{~mm}$, the grid mesh size (distance
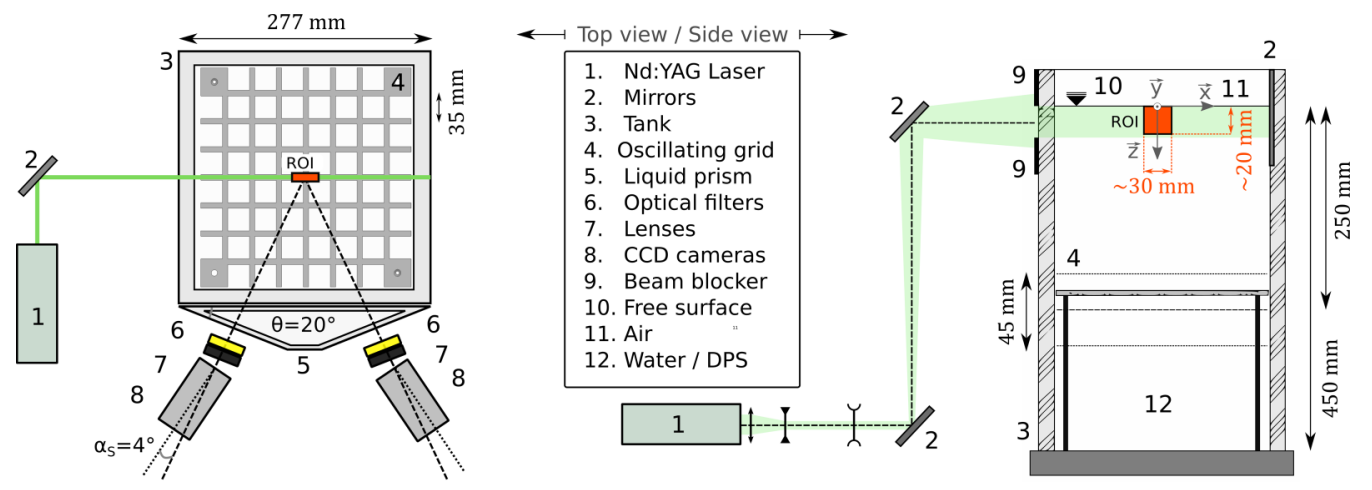

FIG. 1. Sketch of the SPIV setup for liquid phase velocity measurements. 
between the center of two successive grid bars or holes) being $35 \mathrm{~mm}$ (see Fig. 1). This yields a solidity of 0.36 , below the maximum value of 0.4 recommended by Thompson and Turner [39]. The grid-based Reynolds number $\operatorname{Re}_{G}=\rho f S^{2} / \mu$ is equal to 2025 for water and lies in the range of Reynolds numbers explored in the literature [38]). Based on these values and on the power-lawshaped turbulence decay relationships derived by Thompson and Turner [39] or Hopfinger and Toly [40], the velocity and integral length scales could be computed at any distance from the grid in water using the following respective equations:

$$
\begin{gathered}
u^{\prime}=G_{1}(S, M) \times f \times h^{p}, \\
L_{\infty}=G_{2} \times h .
\end{gathered}
$$

However, we consider here the case of shear-thinning, non-Newtonian solutions, for which OGT has never been characterized until recently [38]. Note that previous works on oscillating grid setups operated in viscoelastic solutions exist $[41,42]$. Since turbulence generation is driven by boundary layer interactions at the grid level, and it is known that a minute amount of polymer may significantly modify these boundary layer interactions (drag reduction phenomenon), the properties of OGT in DPS are bound to be different than the water case. A specific particle image velocimetry (PIV) study of the properties of turbulence in the full oscillating grid tank had to be performed for various XG concentrations. In the work published separately, [38], it was found that within the polymer dilute regime of concentrations, turbulence intensity decays as a power-law function of the distance to the grid, as observed by Hopfinger and Toly [40] in water. The power-law exponent $p$ of Eq. (3) varies with polymer concentration, from -0.74 at $10 \mathrm{ppm}$ to -1.18 at $100 \mathrm{ppm}$. The integral length scale is proportional to the grid distance as in water [39], but again with a concentration-dependent slope $G_{2}$ in Eq. (4). Such observations allow one to define, as done for water, undisturbed length and velocity scales of turbulence, but here specific to each polymer concentration used. For the sake of consistency, characteristic turbulence profiles for water measured during this characterization study are used rather than other literature profiles $[39,40]$ in order to estimate $u^{\prime}$ and $L_{\infty}$ for our water experiment. Similar laws are found with a decay exponent for velocity fluctuations of -0.95 . For both water and DPS, good horizontal homogeneity (less than $10 \%$ of turbulence intensity variations along the horizontal dimension $x$ ) is achieved in the central part of the tank, and thus in the central, 30-mm-wide $x$ span corresponding to the ROI for the subsurface study. Vertical turbulent fluctuations are as expected higher than the horizontal ones in the bulk flow, by a factor of 1.1 to 1.4 as expected from OGT experiments in water [3]. However, there are no reasons to believe that this isotropy index should remain identical in the near-surface region, because of the influence of the free surface.

Undisturbed scale values obtained at different polymer concentrations are reported in Table I. The table also shows the corresponding values of the interface Reynolds number based on the maximum viscosity ( $\mu_{0}$ in place of $\mu$ in the Reynolds number definition). Finally, the Deborah numbers based on the shear-thinning timescale $t_{\mathrm{CY}}$ and on the period of grid oscillations are displayed: $\mathrm{De}=t_{\mathrm{CY}} f$. It is a measure of the shear-thinning property of the polymer solution.

The data reported in Table I have to be considered in the light of several important remarks. First, $\operatorname{Re}_{T}$ decreases drastically with increasing polymer concentration, mostly because it is defined on $\mu_{0}$, which may not be a representative viscosity of the flow. When using $\mu_{\infty}$ or the solvent viscosity $\mu_{s}$ with the same length and velocity scales, $\mathrm{Re}_{T}$ values for all polymer concentrations stay approximately within the same range $171 \pm 65$ and $173 \pm 63$, respectively (see the Appendix). A better definition of the turbulent Reynolds number at the interface would be needed for shearthinning fluids. However, even very low values of $\mathrm{Re}_{T}$ are the trademark of a turbulent flow, since this Reynolds number is defined based on turbulent velocity fluctuations and their length scales. The fact that this Reynolds number remains low suggests that the turbulent scale separation may be weak. The results should thus not be used to predicate behaviors for fully developed turbulence, and the conclusions are restricted to low-Reynolds-number, shear-thinning turbulence. 
Second, the undisturbed length scale is estimated by an extrapolation of Eq. (4) at the interface location. This value is not expected to be reached in reality since (1) there is an interface precisely preventing that and (2) confinement effects caused by the tanks wall would also act to modify the integral length scale if it became too large. Yet this concept was used here so as to be consistent with the usual description of oscillating grid turbulence interacting with a free surface.

In fact, in the work of Chiapponi et al. [43], the influence of oscillating grid turbulence in water on the shape and oscillations of a flat free surface is studied, and several domains of interface deformation and motion are evidenced according to the values of undisturbed velocity and length scales. The estimated values for this study are used to check that in this framework, the gas-liquid interface should remain in the "flat" domain in our case. In order to confirm this a priori literature-based hypothesis, posttreatment of recorded particle images is performed so as to isolate the interface location and quantify its motion. It is found that the interface does remain flat in the region of interest, while still showing evidence of a rigid-body up and down-going motion. These oscillations of the free surface have an amplitude between 0.25 and $0.35 \mathrm{~mm}$, decreasing with polymer concentration. It is worth from $1.3 \%$ to $2.5 \%$ of the typical viscous sublayer depth defined in Sec. IV C and corresponds to a maximum of $1.75 \%$ of the depth of the region of interest (ROI). This amplitude defines the uncertainty on the $z=0$ interface location: all profiles plotted versus $z$ are smoothed over a 0.35 -mm-deep span at worst (this thus corresponds to the $z$ uncertainty on all vertical profiles, e.g., in Fig. 3). The most probable origin of such oscillations would not be turbulence, but the minor liquid phase volume variations in the tank caused by the in-and-out motion of rods supporting the grid (sketched in Fig. 1). We can indeed easily estimate the liquid depth variations induced by the displacement of the supporting rods. It is evaluated as $d H=2 \times S \times \pi r_{\text {rod }}^{2} / W^{2}$, where $r_{\text {rod }}$ is the radius of a supporting rod and $W=277 \mathrm{~mm}$ the width of the tank. The value of $d H$ is here $0.26 \mathrm{~mm}$, which compares quite well to the measured oscillations.

\section{Velocity measurements}

Liquid phase velocity measurements in the vicinity of the interface are achieved by stereoscopic particle image velocimetry (SPIV). The experimental setup is sketched in Fig. 1. The ROI is a 20-mm-deep, 30-mm-wide vertical rectangle centered in the tank. The configuration chosen here is that of angular SPIV, with an angle of $\theta=20^{\circ}$. This value was found to be the best compromise between the maximum precision angle of $45^{\circ}$ (as stated by Prasad [44]) and the camera adjustments. A transparent Plexiglas ${ }^{\circledR}$ prism is placed between the cameras and the tank and filled with the same fluid as the one in the tank. The optimal Scheimpflung angle is $\alpha_{s}=4^{\circ}$. The cameras used are double-frame PCO CCD sensors of $1280 \times 1024$ pixels. Both are equipped with a $60 \mathrm{~mm}$ focal macro lens. A pulsed Quantel Nd:YAG laser emitting at a $\lambda=532 \mathrm{~nm}$ wavelength is used to illuminate hollow glass sphere particles of $10 \mu \mathrm{m}$ nominal diameter. The seeding particle density closely matches that of the working fluid. The particle Stokes number based on either integral or Kolmogorov scales are always lower than $10^{-3}$, thus confirming that particles are good flow tracers. The laser sheet thickness achieved is of about $250 \mu \mathrm{m}$. Reflecting, nonfluorescent particles were chosen in order to ensure the possibility of a further coupling of SPIV with fluorescencebased methods (PLIF) for near-surface mixing measurements. For PLIF or (S)PIV measurement, reflections at the gas-liquid interface can alter the homogeneity of illumination of the ROI and thus the quality of measurement. Robust methods have been developed to account for this effect [45]. Yet they were not required here: as the interface remained flat, reflections stayed localized in time and could be easily accounted for by a simple image normalization process. The SPIV is performed in double-frame mode. The interval between two frames is set at $d t_{\mathrm{SPIV}}=12 \mathrm{~ms}$. The acquisition frequency is $f_{\text {acq }}=4 \mathrm{~Hz}$, and the time between two successive instantaneous vector fields is thus $250 \mathrm{~ms}$. Based on the estimated velocity and length scales, the velocity measurements are thus not time resolved. The \#f numbers of cameras 1 and 2 are set, respectively, at 16 and 11 . Theses two 

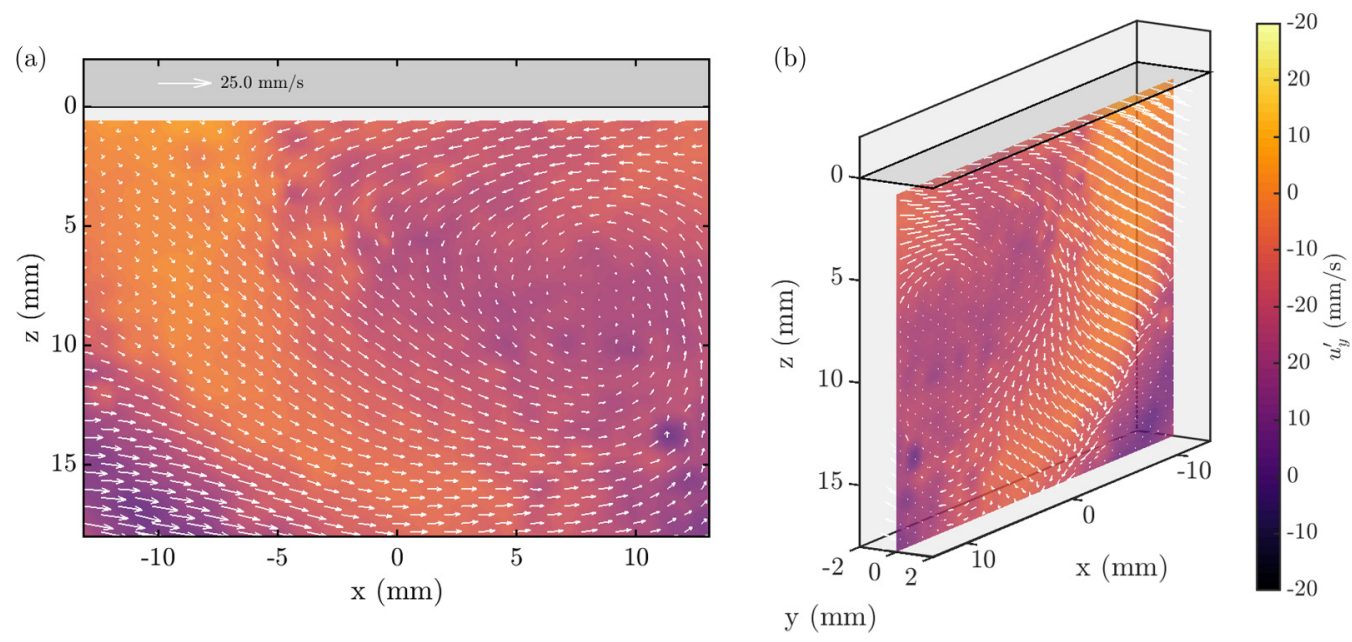

FIG. 2. Example of velocity fluctuation snapshot fields $u^{\prime}$ for water in 2D2C $[x-z$ plane (a) $]$ and 2D3C (b). The colorbar represents the out-of-plane velocity component $u_{y}^{\prime}$. Only one of every four vectors is shown in both the $x$ and $z$ dimensions for the sake of readability.

numbers are different to account for the fact that camera 2 collects more light than camera 1 since it faces the illumination setup.

Vector fields are computed with DaVis 8 software using a multipass processing: a first pass with $64 \times 64$ pixels and two following passes with $32 \times 32$ pixels, round-shaped, Gaussian weighted interrogation windows, at a maximum $50 \%$ overlap. The final spatial resolution achieved is $0.34 \mathrm{~mm}$. Spurious vectors are removed from PIV fields by applying a threshold of 1.2 on the peak ratio and replaced using median filtering. Uncertainties on SPIV velocity measurements are estimated with the DaVis software according to the method of Wieneke [46]. Thorough analysis has been made for one water run and yields the following typical errors: $\Delta U_{x}=0.2 \mathrm{~mm} / \mathrm{s}$, $\Delta U_{y}=0.4 \mathrm{~mm} / \mathrm{s}$, and $\Delta U_{z}=0.1 \mathrm{~mm} / \mathrm{s}$. The uncertainty on the out-of-plane velocity component $U_{y}$ is higher than on the in-plane components because of the stereoscopic reconstruction step. The stereo reconstruction error evaluated in the same way is typically of $0.15 \mathrm{px}$.

Oscillations of the grid are started at least 30 min before the beginning of PIV measurements, from a fluid initially at rest. Ten thousand vector fields are recorded for each run, corresponding to a measurement time of $2500 \mathrm{~s}$ (approx. $40 \mathrm{~min}$ ) at $f_{\text {acq }}=4 \mathrm{~Hz}$. Integral timescales of turbulence can be estimated under the interface, by dividing for each $z$ the integral length scales by the corresponding velocity scale at the same depth (both available in Fig. 3). The highest value of the integral timescale along $z$ is noted $\tau_{L}$. It is checked that for each working fluid, the duration of measurement is at least $170 \times \tau_{L}$, that is, that the statistical analysis of velocities is performed over at least 170 uncorrelated large-scale events.

\section{RESULTS}

\section{A. Hydrodynamics in the surface-influenced layer}

From the set of instantaneous fields acquired, it is possible to compute an ensemble average, corresponding to the mean flow under the interface. Turbulent fluctuations are then deduced by subtracting this mean flow from instantaneous fields. An example of fluctuating velocity fields for water is displayed in Fig. 2. As expected from the three-dimensional nature of the flow, several types of fluid motions are combined: lateral sweeping, up or-down going motion, and swirling structures. SPIV allows one to make out the out-of-plane horizontal features of instantaneous eddies 

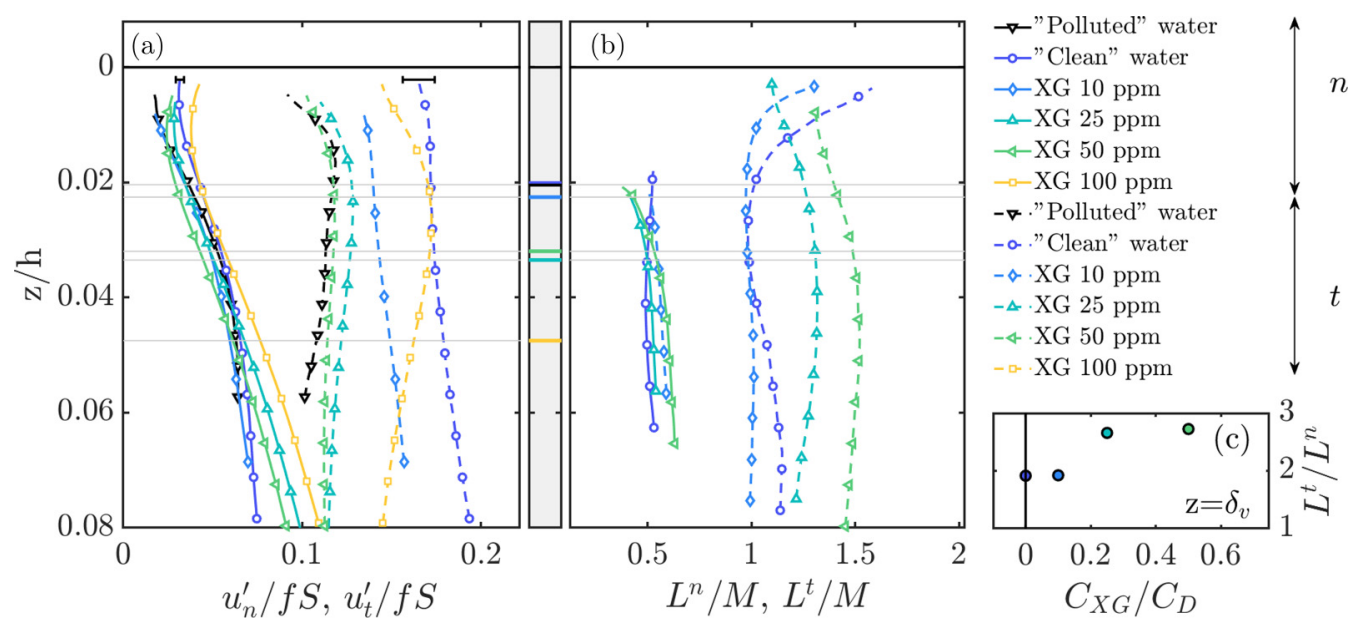

FIG. 3. (a) Profiles of normal and tangential turbulent rms velocities $u_{n}^{\prime}$ (full lines) and $u_{t}^{\prime}$ (dashed lines) along $z / h$, scaled by a grid reference velocity $f S$. Typical uncertainties on velocity values are shown for the clean water run near the interface with black error bars. $h$ is the distance between the average grid position and the surface. (b) Profiles of vertical/normal integral length scale $L^{n}$ (full lines) and horizontal/tangential integral length scale $L^{t}$ (dashed lines) along $z / h$, scaled by the grid mesh parameter $M$. Markers in (a) and (b) are plotted on one of every five data points for the sake of readability. For $100 \mathrm{ppm}$, length scales become much larger than the ROI, which prevents the integral length-scale computation. Horizontal thin gray lines are plotted at each viscous sublayer depths reported, and colored ticks on the central bar indicate which working fluid they correspond to. (c) Evolution of $L^{t} / L^{n}$ with $C_{\mathrm{XG}} / C_{D}$ at $\mathrm{z}=\delta_{v}$.

reaching the interface, by taking a planar slice of the three components' velocity field, which is not accessible through classical PIV. Based on the estimation of the undisturbed integral length scale values $L_{\infty}$ shown in Table I, and under the hypothesis that the surface-influenced layer has a depth of approximately $L_{\infty}$, one can assume that the present ROI is included within the surface-influenced layer.

In order to check whether the energy transfer concept is still valid in DPS, the width-averaged profiles of the root mean square (rms) of vertical and horizontal velocity fluctuations are plotted in Fig. 3(a). The vertical fluctuation rms profile, based on velocities normal to the interface (along $z$ ), is defined as $u_{n}^{\prime}=\left[\left\langle u_{z}^{\prime}\right\rangle_{\mathrm{rms}}\right]_{x},\langle\cdot\rangle_{\mathrm{rms}}$ denoting a rms operator over the full set of data, and $[\cdot]_{x}$ an horizontal averaging. The horizontal fluctuation rms profile, based on velocities tangential to the interface (along $x$ and $y$ ), is then defined as $u_{t}^{\prime}=\left[\sqrt{\left\langle u_{x}^{\prime}\right\rangle_{\mathrm{rms}}^{2}+\left\langle u_{y}^{\prime}\right\rangle_{\mathrm{rms}}^{2}}\right]_{x}$. Using SPIV data, horizontal isotropy can be checked showing that $\left\langle u_{x}^{\prime}\right\rangle_{\mathrm{rms}}$ and $\left\langle u_{y}^{\prime}\right\rangle_{\mathrm{rms}}$ mappings are similar.

The integral length scales of turbulence $L_{i j}^{k}$ are defined as the integral of correlation coefficients of velocity fluctuations components $i$ and $j$ along dimension $k$, with $i$, jor $k=x, y$, or $z$.

Computed length scales are averaged over sampling regions at different depths. Length scales along $x$ are computed on sampling regions as wide as the ROI's and including three vectors in the $z$ direction. Length scales along $z$ are computed on sampling regions of width equal to that of the ROI's and $10 \mathrm{~mm}$ height. As will be observed later, values of integral length scales may be of the same order of magnitude as the size of the sampling region. In that case the integral of the correlation coefficient may not be converged. To account for that, correlation coefficient curves are fitted by a Gaussian function, and the length scales are computed as the integral from zero to infinity of this function (rather than the integral of the correlation data). This fitting procedure proved to be efficient for every case except the $100 \mathrm{ppm}$ concentration one, for which the integral length scales are much larger than the size of the ROI and cannot be accessed at all. The vertical/normal 
and horizontal/tangential integral length scales are finally defined according to Janzen et al. [1], respectively, as

$$
\begin{aligned}
& L^{n}=\sqrt{\left(L_{x x}^{z}\right)^{2}+\left(L_{y y}^{z}\right)^{2}+\left(L_{z z}^{x}\right)^{z}}, \\
& L^{t}=\sqrt{\left(L_{x x}^{x}\right)^{2}+\left(L_{y y}^{x}\right)^{2}+\left(L_{z z}^{x}\right)^{2}},
\end{aligned}
$$

and their profiles along depth are shown in Fig. 3. The typical uncertainty on $L^{n}$ or $L^{t}$ can be estimated by uncertainty propagation of the $95 \%$ confidence intervals for the Gaussian fitting parameters and is found to be of about $15 \%$.

In the surface-influenced layer, the horizontal velocity fluctuations are stronger than the vertical ones. This is verified for all water and DPS runs no matter the concentration. Moreover, the vertical fluctuations strongly decrease when approaching the interface, whereas horizontal fluctuations only weakly decrease, stay approximately constant, or even increase. Turbulence coming from the oscillating grid is more energetic in the vertical direction due to the grid motion and decays when moving away from the grid. Here inside the blocking layer, the vertical ones decay much faster than the horizontal ones and are lower. Their energy has been and is being transferred to the horizontal ones, leading to an inversion of the dominant components (vertical/normal one in the bulk versus horizontal/tangential one in the surface-influenced layer). The decay of horizontal/tangential turbulence intensity is balanced, which may even lead it to increase [4,5].

As for the length scales it appears that the horizontal ones always larger than vertical ones. This has already been evidenced for water by Janzen et al. [1] and explained as the consequence of intercomponent exchanges for turbulence approaching the interface: eddies become "flat" and tend to stretch along horizontal dimensions. Such a behavior is here also observed for DPS at all studied concentrations. The horizontal "stretching" of turbulent structures even seems to be amplified by the presence of s polymer: the $L^{t} / L^{n}$ ratio at $\mathrm{z}=\delta_{v}$ jumps from 2 to 2.7 between 10 and $25 \mathrm{ppm}$ [Fig. 3(c)].

However, this does not necessarily mean that the energy transfer mechanism itself is enhanced by the presence of polymer: indeed, looking again at Fig. 3, it appears that the ratio between $u_{t}^{\prime}$ and $u_{n}^{\prime}$ mostly decreases with increasing polymer concentration. These seemingly contradictory observations suggest a more complex energy transfer mechanisms for the polymer case. This last statement is supported by the observation of Fig. 4(a), where the quantities of Fig. 3 are plotted normalized by the undisturbed scale values for each working fluid. In water, $u_{t}^{\prime} / u^{\prime}$ is close to unity, and $u_{n}^{\prime} / u^{\prime}$ slightly below, as a consequence of the energy transfer mechanism. Yet for polymer experiments, a striking underprediction of both vertical and horizontal turbulence intensity in the region of interest by the undisturbed velocity scale is witnessed. It is not in itself unexpected that $u^{\prime}$ underpredicts horizontal turbulence intensity at the interface, even for water: $u^{\prime}$ comes from an input turbulence where it is intrinsically lower than the vertical velocity component, but the energy transfer mechanism is such that it is enhanced while approaching the interface. The energy transfer balancing the inherent turbulent intensity decay can thus lead to an apparent increase in horizontal turbulence intensity. To that extent, vertical turbulence intensity being in the bulk intrinsically higher (because of the vertical grid oscillations), but decreased by energy transfer mechanisms in the surface-influenced layer, it should fall slightly below the $u^{\prime}$ prediction, as observed here for both water runs.

Yet, for polymer cases, both $u_{t}^{\prime}$ and $u_{n}^{\prime}$ appear higher than $u^{\prime}$, at least at the highest $z / L_{\infty}$. On can compare between a measured turbulent kinetic energy scale $k_{m}$, computed as $k_{m}=\sqrt{\left(u_{n}^{\prime}\right)^{2}+2\left(u_{t}^{\prime}\right)^{2}}$, and an undisturbed turbulent kinetic energy scale estimated from $u^{\prime}$ as $k_{u}=\sqrt{\left(\beta u^{\prime}\right)^{2}+2\left(u^{\prime}\right)^{2}}$ with $\beta$ between 1.1 and 1.4 (see Sec. III B). At $z=\delta_{v}$, measured kinetic energy profiles $k_{m}$ reach values up to four times higher than the undisturbed prediction $k_{u}$. The $k_{m} / k_{u}$ quantities increase with increasing polymer concentration, suggesting that this underprediction is correlated to polymer 

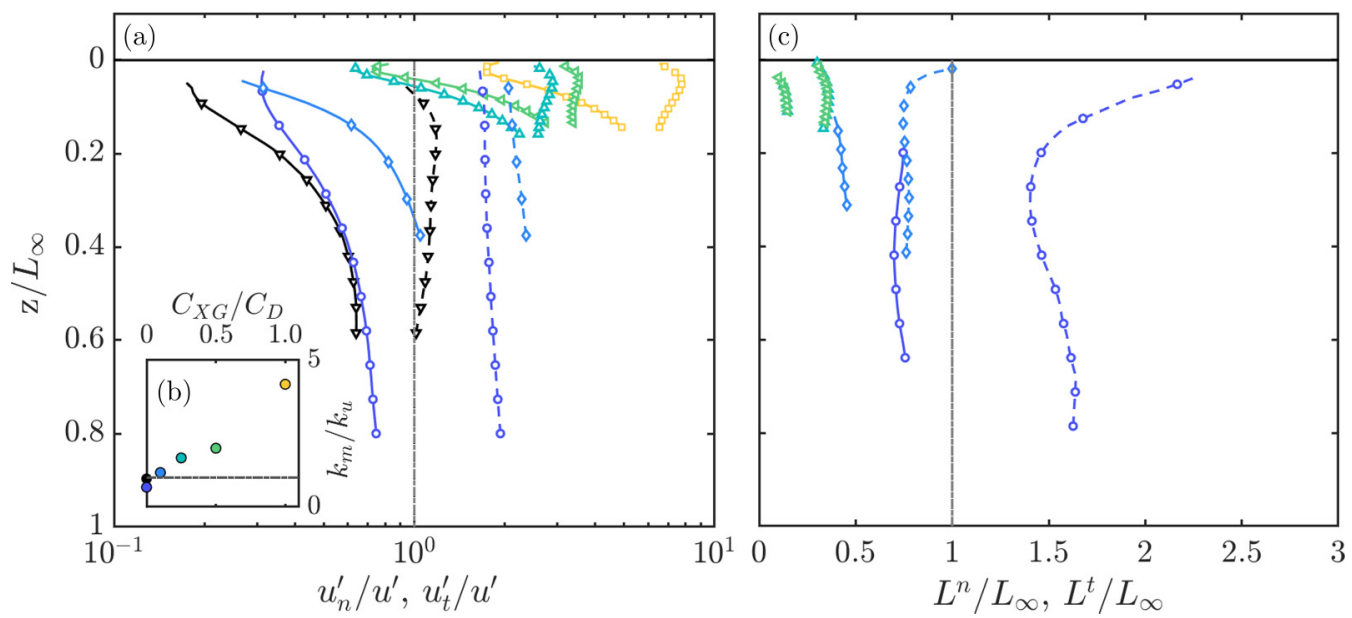

FIG. 4. (a) Profiles of normal and tangential turbulent rms velocities $u_{n}^{\prime}$ (full lines) and $u_{t}^{\prime}$ (dashed lines) normalized by the undisturbed velocity scale (based on horizontal velocity fluctuations) $u^{\prime}$, along the depth $z$ itself normalized by the undisturbed integral scale $\mathrm{E}_{\infty}$. The dashed-doted vertical gray line shows is plotted at $u_{n}^{\prime}=u^{\prime}$ or $u_{t}^{\prime}=u^{\prime}$. (b) Evolution of the measured kinetic energy $k_{m}$ at $z=\delta_{v}$ divided by the predicted, undisturbed, kinetic energy $k_{u}$, as a function of polymer concentration, the dashed-doted line is plotted at $k_{u}=k_{m}$. Markers in (a) and (b) are plotted on one of every five data points for the sake of readability. (c) Profiles of normal an tangential integral length scales $L^{n}$ and $L^{t}$ normalized by $L_{\infty}$ plotted versus $z \mathrm{E}_{\text {infty }}$. The color code is the same as in Fig. 3.

effects [Fig. 4(b)]. In Fig. 4(c) it appears that the two $L^{i} / L_{\infty}$ curves for $C_{\mathrm{XG}}>10 \mathrm{ppm}$ collapse, while this is not the case for lower concentrations.

As a conclusion, the general concept of energy transfer from horizontal to vertical velocity components is verified in water and seemingly remains valid in DPS. The presence of polymer apparently enhances the stretching of turbulent structures' length scales in the surface-influenced layer, but the overall energy transfer mechanism in the surface-influenced layer seems more complex in DPS, and evolving with polymer concentration.

One should qualify the previous conclusions, by reminding that the structure of upcoming turbulence varies with polymer concentration, as well as its intensity $\left(\operatorname{Re}_{T}\right.$ when defined on zero shear viscosity). The influence of polymer concentration has to be considered in the light of the low-Reynolds-number range investigated and its variation with polymer concentration. Further studies on this mechanisms should thus focus on defining precisely the surface-influenced layer's depth, which might be different than $L_{\infty}$ in the polymer case, but also on providing a clear insight on the distinct effect of the Reynolds number in shear-thinning polymer solutions.

\section{B. Horizontal motion in the viscous sublayer}

In Fig. 3, two different types of evolution for $u_{t}^{\prime}$ approaching the interface are possible. For "clean" water and $10 \mathrm{ppm}$ cases, $u_{t}^{\prime}$ weakly decreases with decreasing z. For other DPS cases and "polluted" water, it increases and exhibits a peak at depths between 5 and $10 \mathrm{~mm}$. This peak is, by definition, located just above the viscous sublayer depth, marked by horizontal lines on the figure (see the next section for their definition). Regarding the evolution of integral length scales along depths in Fig. 3(b), results for water are consistent with the trends found by Janzen et al. [1]: The vertical length scale is globally constant at the depths considered. The horizontal length scale is also nearly constant except inside the viscous sublayer where it increases. A similar evolution is observed for the $10 \mathrm{ppm}$ DPS. The trend is, however, quite different for 25 and $50 \mathrm{ppm}$ concentrations: the vertical scale slightly decreases when approaching the interface, and the increase of horizontal scales 


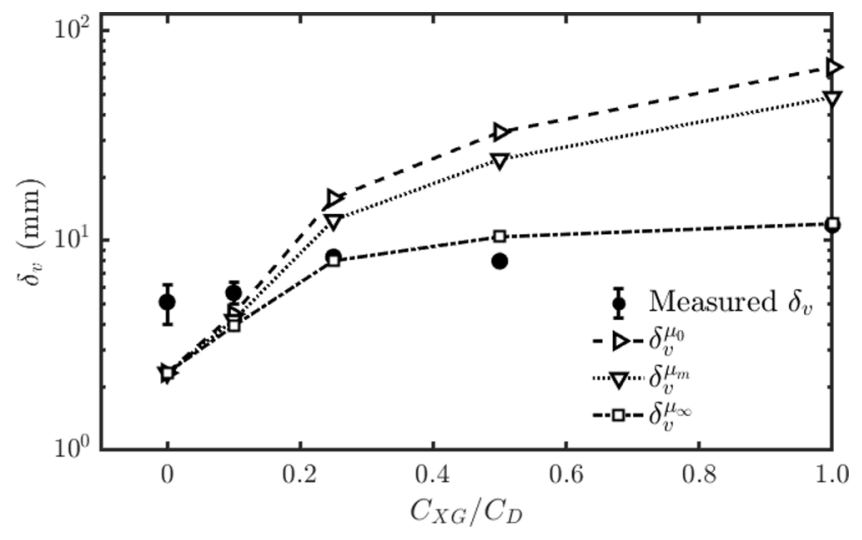

FIG. 5. Evolution of the measured viscous sublayer depth $\delta_{v}$ (log scale, black dots) with scaled polymer concentration compared to predictions based on undisturbed scales and constant scale viscosities $\mu_{0}$ (rightpointing triangles), $\mu_{m}=\left(\mu_{0}+\mu_{\infty}\right) / 2$ (down-pointing triangles), and $\mu_{\infty}$ (squares).

disappears to be replaced by a slow decrease with decreasing $z$. These results seem to indicate that inside the viscous sublayer, another mechanism related to horizontal/tangential velocity fluctuations is added when polymer or surfactants are introduced. Its nature is discussed in the next section. It can be emphasized that the present measurements have been performed at a higher spatial resolution than the existing literature experiments: at least six points of measurement are obtained inside the viscous sublayer for the run with the smallest $\delta_{v}$. They are also the only local measurements of flow properties in shear-free viscous sublayers of dilute polymer solutions.

\section{Viscous sublayer depth}

According to Hunt [4] and Brumley and Jirka [5], the viscous sublayer depth can be measured by computing the gradient along $z$ of tangential velocity fluctuations rms profiles, and finding the depth at which it is minimum (maximal negative amplitude, noting that $z$ is oriented downwards). In other words, the viscous sublayer depth is found where the tangential velocity fluctuations increase rate is the highest as approaching the interface [5]. Indeed, entering the viscous sublayer, energy transfer mechanisms occurring in the surface-influenced layer are balanced by viscous mechanisms. The increase on tangential velocity fluctuations induced by energy transfer is limited by viscous damping, thus leading to a inversion of the previous gradient [4].

Measured depths are indicated by horizontal lines on Fig. 3. The evolution of $\delta_{v}$ with $C_{\mathrm{XG}}$ is shown in Fig. 5. For water, $\delta_{v}$ is about twice the prediction of Eq. (1). This is consistent with the latest result of Variano and Cowen [2] and with the use of the prefactor 2 in Eq. (1) in some works [16]. In DPS it appears that $\delta_{v}$ globally increases with increasing polymer concentration. The question is whether this increase is due to the increase in overall viscosity, the apparent decrease of $\mathrm{Re}_{T}$, or another factor. To investigate this point, measurements of $\delta_{v}$ are compared to predictions $\delta_{v}^{\mu_{i}}$ based on Eq. (1). For these predictions the inputs are the concentration-dependent undisturbed scale $L_{\infty}$ and $u^{\prime}$, and the interfacial Reynolds number computed using $u^{\prime}, L_{\infty}$ along with three scale viscosities $\mu_{i}$,

$$
\delta_{v}^{\mu_{i}}=L_{\infty}\left(\frac{\rho u^{\prime} L_{\infty}}{\mu_{i}}\right)^{-0.5}
$$

such that $\mu_{i}=\left[\mu_{\infty}, \mu_{m}=\frac{\mu_{\infty}+\mu_{0}}{2}, \mu_{0}\right]$. It appears that these "Newtonian-like" predictions, when based on zero shear-rate viscosity $\mu_{0}$ or intermediate viscosity $\mu_{m}$, overpredict the increase of $\delta_{v}$ for $C_{\mathrm{XG}} / C_{D}>0.1$. The closest modeling is achieved for $\delta_{v}^{\mu_{\infty}}$, that is, Eq. (7), using infinite 
shear-rate viscosity. It is quite surprising since the typical average shear-rate magnitudes achieved under the interface are of about $1 \mathrm{~s}^{-1}$, far from the $10^{3} \mathrm{~s}^{-1}$ order of magnitude required by the CY-like shear-thinning curve to reach $\mu_{\infty}$. Yet instantaneous local shear-rate values may be much higher whenever high-vorticity turbulent structures reach the interface. Starting from Eq. (1) and taking into account the variations of undisturbed scales induced by the presence of polymer, the evolution of a viscous sublayer with polymer concentration can be modeled, based on rheological concentration-dependent parameters of polymer solutions. This is discussed in the following section.

\section{DISCUSSION}

\section{A. Modeling of the viscous sublayer depth}

In this section, attempt is made to determine some trends on the dependency of the viscous sublayer depth $\delta_{v}$ in shear-thinning DPS, as a function of the Deborah number De and Reynolds number, viscous sublayer depth in water $\delta_{v}^{w}$, and Reynolds number in water $\operatorname{Re}_{T}^{w}$ as a limiting case for very dilute polymer solutions $(\mathrm{De}=0)$. The $w$ superscript used hereinafter denotes the values of scales (velocity, length scales) and other parameters (Reynolds number, constants, viscosity, etc.) in water. Starting from the "Newtonian," waterlike scaling for the viscous sublayer depth, defined by Eq. (1), one can as a first approach extend the scaling to the following relationship for $\delta_{v}$ :

$$
\delta_{v}=K L_{\infty} \operatorname{Re}_{T}^{-e},
$$

where $K$ is an empirical constant. For water, $e=-0.5$ and $K=K^{w}$ is close to 2 as observed in this work or in that of Variano and Cowen [2]. Note that in the reference model of Eq. (1) [5], $K=K^{w}$ is equal to one. The effect of variable viscosity can be introduced in the expression of the Reynolds number by writing that

$$
\operatorname{Re}_{T}=\frac{\rho u^{\prime} L_{\infty}}{\mu}=\frac{\rho u^{\prime w} L_{\infty}^{w}}{\mu^{w}} \times \frac{u^{\prime}}{u^{\prime w}} \times \frac{L_{\infty}}{L_{\infty}^{w}} \times \frac{\mu^{w}}{\mu}=\operatorname{Re}_{T}^{w} \times \frac{u^{\prime}}{u^{\prime w}} \times \frac{L_{\infty}}{L_{\infty}^{w}} \times \frac{\mu^{w}}{\mu},
$$

assuming that the density does not change with the presence of polymer. Replacing $\operatorname{Re}_{T}$ by the previous expression in Eq. (8), writing $L_{\infty}$ as $L_{\infty}^{w} \times L_{\infty} / L_{\infty}^{w}$ and $K$ as $K / K^{w} \times K^{w}$, and simplifying, one gets

$$
\delta_{v}=\frac{K}{K^{w}} \times K^{w} \times L_{\infty}^{w} \times\left(\operatorname{Re}_{T}^{w}\right)^{-e}\left(\frac{L_{\infty}}{L_{\infty}^{w}}\right)^{1-e} \times\left(\frac{u^{\prime}}{u^{\prime w}}\right)^{-e} \times\left(\frac{\mu}{\mu^{w}}\right)^{e} .
$$

In Eq. (10), the power law of the Reynolds number can be decomposed as $\left(\operatorname{Re}_{T}^{w}\right)^{-e}=$ $\left(\operatorname{Re}_{T}^{w}\right)^{-e+0.5} \times\left(\operatorname{Re}_{T}^{w}\right)^{-0.5}$. In doing so, the $\left(\operatorname{Re}_{T}^{w}\right)^{-0.5}$ trend characteristic of water appears, and one may use the $K^{w} \times L_{\infty}^{w}$ remaining term and Eq. (1) to write that $\delta_{v}^{w}=K^{w} \times L_{\infty}^{w} \times\left(\operatorname{Re}_{T}^{w}\right)^{-0.5}$ and to reshape Eq. (10) as

$$
\frac{\delta_{v}}{\delta_{v}^{w}}=\frac{K}{K^{w}} \times\left(\operatorname{Re}_{T}^{w}\right)^{-e+0.5} \times\left(\frac{u^{\prime}}{u^{\prime w}}\right)^{-e} \times\left(\frac{L_{\infty}}{L_{\infty}^{w}}\right)^{1-e} \times\left(\frac{\mu}{\mu^{w}}\right)^{e} .
$$

This expression describes the increase in viscous sublayer depth upon polymer addition based only on a well-defined Reynolds number for water, upcoming turbulence parameters described by the undisturbed scales, and effective shear-thinning viscosity $\mu$. The viscosity term $\left(\frac{\mu}{\mu^{w}}\right)$ can be described by the shear-thinning CY model. Isolating the effective viscosity $\mu$ in Eq. (2), dividing by $\mu^{w}$, and using the fact that for all polymer concentrations in the dilute regime we have $\mu_{\infty} \simeq \mu^{w}$, one gets

$$
\frac{\mu}{\mu^{w}}=1+\left(\frac{\mu}{\mu_{0}}-1\right)\left[1+\left(t_{\mathrm{CY}} \dot{\gamma}\right)^{a}\right]^{(n-1) / a}
$$



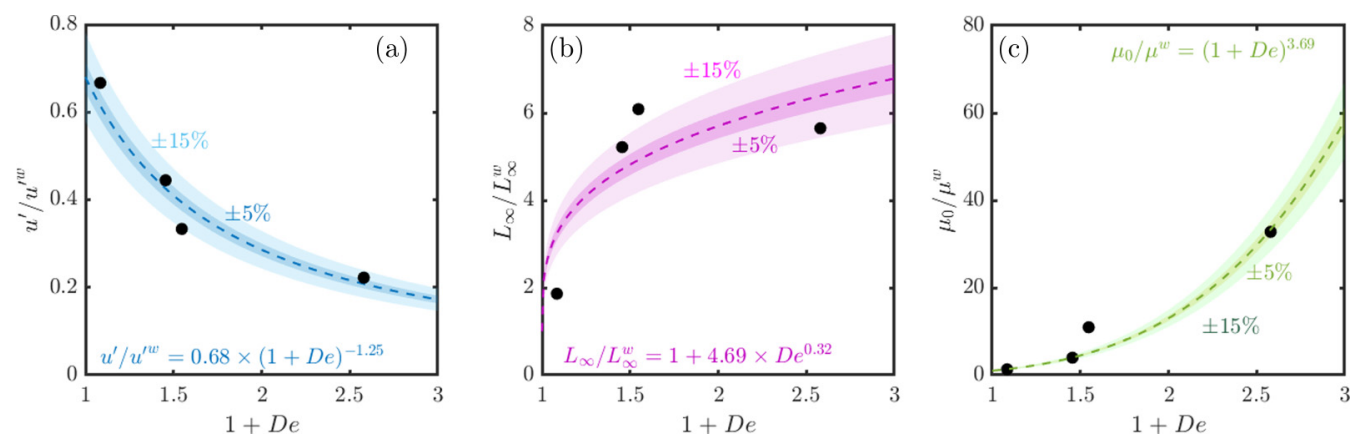

FIG. 6. Modeling of terms A (a), B (b), and C (c) in terms of grid-based Deborah number. A represents the ratio of undisturbed velocity scale in polymer over that for water. $\mathrm{B}$ is the ratio of undisturbed integral scales, and $C$ the ratio between zero shear rate (maximum) viscosity and viscosity of water. Scaling laws are obtained by least-square fitting and shown on each associated plot. Shaded areas represent scaling uncertainty areas of, respectively, $\pm 5 \%$ for the darker and $\pm 15 \%$ for the lighter.

For the working fluids considered here, one may take $a=2$ and $n \simeq 0.55$ (see Table I), and so $(n-1) / a=-0.225$. The effective subsurface shear rate $\dot{\gamma}$ can be related to the grid sweep frequency writing that $\dot{\gamma}=K_{s} \times f$. In doing so, the definition of the grid-based Deborah number appears in Eq. (12), which can be rewritten as

$$
\frac{\mu}{\mu^{w}}=1+\left(\frac{\mu}{\mu_{0}}-1\right)\left[1+\left(K_{s} \mathrm{De}\right)^{2}\right]^{-0.225} .
$$

Finally, using Eqs. (11) and (13), it comes that

$$
\frac{\delta_{v}}{\delta_{v}^{w}}=\frac{K}{K^{w}} \times\left(\operatorname{Re}_{T}^{w}\right)^{-e+0.5} \times\left(\frac{u^{\prime}}{u^{\prime w}}\right)^{-e} \times\left(\frac{L_{\infty}}{L_{\infty}^{w}}\right)^{1-e} \times\left\{1+\left(\frac{\mu}{\mu_{0}}-1\right)\left[1+\left(K_{s} \mathrm{De}\right)^{2}\right]^{-0.225}\right\}^{e} .
$$

The value of $K_{s}$ is used to define the relationship between the forcing shear rate at the grid level and the effective shear rate under the interface. Modelling the evolution of the viscous sublayer depth with shear thinning comes to modeling the evolution of terms $A=\frac{u^{\prime}}{u^{\prime w}}, B=\frac{L_{\infty}}{L_{\infty}^{w}}$, and $C=$ $\frac{\mu_{0}}{\mu^{w}}$ with a parameter representative of the shear-thinning property. Here the grid-based Deborah number previously defined is used. Contrary to the turbulent interfacial Reynolds number $\operatorname{Re}_{T}$ which requires the definition of a reference scale viscosity, the definition of the grid-based Deborah number is unambiguous and based solely on polymer properties and a constant bulk turbulence parameter, $f$. Data points for $A, B$, and $C$ are deduced from the values of $L_{\infty}, u^{\prime}$, and $\mu_{0}$ reported in Table I and plotted versus $(1+\mathrm{De})$ in Figs. 6(a)-6(c).

For the sake of consistency, all ratios should tend to unity when De tends to zero, since the zero Deborah case corresponds to water. Following our results, power laws could be well adapted to reproduce the De dependency of $A, B$, and $C$. Applying a least-square fitting method allows us to determine the best power-law exponent. Increase or decrease of $A$ and $C$ term are well modeled by, respectively, $A=K_{A}(1+\mathrm{De})^{\alpha}$ and $C=K_{C}(1+\mathrm{De})^{\kappa}$ with $K_{A}=0.68, \alpha=-1.25, K_{C}=1$, and $\kappa=3.69$. The increasing trend of $B$ is best approximated by an expression of type $B=1+K_{B} \mathrm{De}^{\beta}$, with $K_{B}=4.69$ and $\beta=0.32$. This different shape allows us to account for the reduced increase rate of $B$ at De $>0.4$.

From the observations of Sec. IV C, the best scaling of the viscous sublayer depth comes from an apparent infinite shear rate estimation. An infinite shear rate can be modeled by setting $K_{s}$ at an infinite value. This comes to a ratio $\mu / \mu^{w}$ that tends to unity since it has been previously assumed 


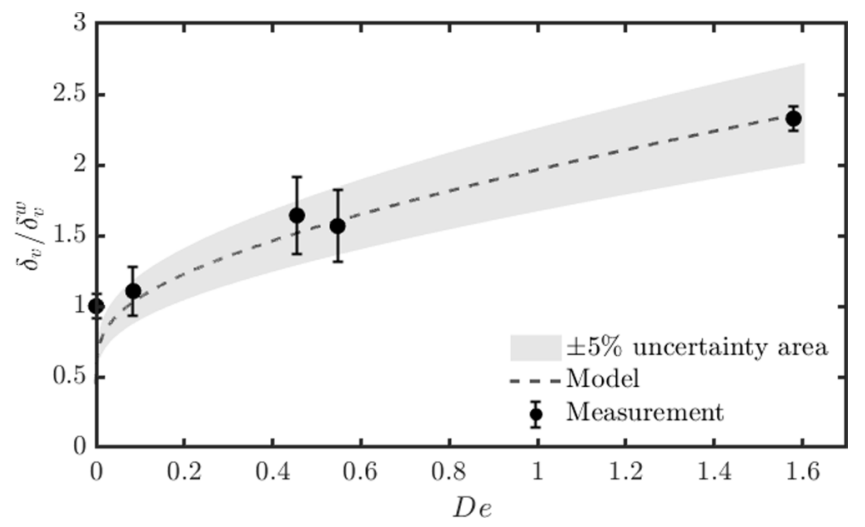

FIG. 7. Modeling of the viscous sublayer depth using Eq. (16). Black dots are measured points. The error bars on measured $\delta_{v} / \delta_{v}^{w}$ are deduced by adding relative uncertainty on $\delta_{v}$ for each polymer case to that for water. The shaded region corresponds to $\pm 5 \%$ uncertainty area on the model.

that $\mu^{w} \sim \mu_{\infty}$. Equation (11) or (14) (equivalent in that case) thus becomes

$$
\frac{\delta_{v}}{\delta_{v}^{w}}=\frac{K}{K^{w}} \times\left(\operatorname{Re}_{T}^{w}\right)^{-e+0.5} \times\left(\frac{u^{\prime}}{u^{\prime}}\right)^{-e} \times\left(\frac{L_{\infty}}{L_{\infty}^{w}}\right)^{1-e} .
$$

Following our results for $\delta_{v}$ at different $C_{\mathrm{XG}}$ concentrations, one gets $K=0.32$ and $e=0.29$. The final expression for the model curve is then

$$
\frac{\delta_{v}}{\delta_{v}^{w}}=0.16 \times\left(\operatorname{Re}_{T}^{w}\right)^{0.21} \times\left[0.68(1+\mathrm{De})^{-1.25}\right]^{-0.29} \times\left(1+4.69 \mathrm{De}^{0.32}\right)^{0.71} .
$$

This resulting relationship is plotted in Fig. 7 and compared to measured depths. With that approach, the influence of polymer is introduced by the variations of scales coming from the bulk turbulence (which are not related to surface mechanisms) but also by the $e=0.29$ exponent, which differs from its 0.5 value in water. Moreover, the efficiency of the infinite shear rate and minimum viscosity assumption at the interface are confirmed. The increase in the viscous sublayer depth can thus not only be attributed to an increase in overall apparent viscosity. It involves viscous and shear-thinning effects and comes not only from a modification of the undisturbed scales, but also from and surface-associated mechanisms expressed through the value of $e$.

The accuracy of the model has to be moderated by the small number of data points available. The main difficulty of adding data points comes from the fact that the polymer dilute regime corresponds to a narrow range of grid-based Deborah number variations. In order to complete the previous trend, one could perform additional measurements at intermediate polymer concentrations between 10 and $100 \mathrm{ppm}$, or try to explore the highly dilute regime for $C_{\mathrm{XG}}<10 \mathrm{ppm}$. Another way would be to vary grid frequency at fixed polymer concentration, but this would also result in variations on the Reynolds number and undisturbed scales.

\section{B. Damping of horizontal fluctuations at the interface}

As previously mentioned, an important factor affecting the behavior of turbulence approaching a gas-liquid interface is the cleanliness of this interface. Clean surfaces allow horizontal velocity fluctuations at the very limit between the gas and the liquid. On the other hand, when a free surface is covered by organic or surface active material (surfactants), the rheological properties of these materials may be such that they creates a tangential stress and damps turbulent velocity fluctuations $[9,47]$. From a hydrodynamic point of view, turbulence close to highly polluted interfaces is still different from that of turbulence close to a rigid wall [9]. 
Here it appears from the shape of the RMS profiles in Fig. 3 that additional horizontal stresses are introduced by the polymer for $C_{\mathrm{XG}}>10 \mathrm{ppm}$ in the dilute regime, as in the polluted water case. Yet at those concentrations, the surface tension of XG solutions is not significantly different from that of water $[48,49]$. XG is thus not a surfactant. This indicates that horizontal stresses observed are induced by shear thinning. In other words, the influence of shear thinning may "look like" that of surface activity in terms of hydrodynamics.

Once again, additional investigation on the effect of the Reynolds number would be needed in order to generalize the present conclusion and discard any effects due to turbulent intensity. The comparison of the surface tension dynamics with the shear-thinning timescale $t_{C Y}$ would also bring further information and allow us to quantify the dynamical effects of the polymer at the interface in terms of surface tension versus shear-thinning properties. This point is crucial to further mass transfer studies: the presence of a surfactant is known to have a great impact on mass transfer $[8,50]$, and it should be the same for shear-thinning polymers.

\section{Critical polymer concentration}

From $C_{\mathrm{XG}}=25 \mathrm{ppm}$ and above, horizontal damping mechanisms are evidenced. There should thus exist a critical polymer concentration between 10 and $25 \mathrm{ppm}$ marking the onset of the horizontal damping. Quite interestingly, Wyatt et al. [35] evidence drag reduction for XG flowing in pipes at XG concentrations down to $20 \mathrm{ppm}$. Their critical drag reduction concentration is around $70 \mathrm{ppm}$. It tends to indicate that even within the dilute regime, several subregimes for hydrodynamics and mass transfer exist. It is worth noting that the existence of such inner dilute regimes was already evidenced for viscoelastic dilute solutions of other polymer molecules [41,51].

The origin of the influence of polymer concentration should find an explanation in polymer-flow and polymer-polymer interactions: here in the dilute regime, it is assumed that polymer chains do not interact with each other, but only with the flow. Yet two distinct hydrodynamic behavior are evidenced. An appropriate comparison of polymer typical size and molecular relaxation timescales with the Kolmogorov timescale of the flow could help further understanding of these subdilute critical concentration, and extend results to different polymer molecules of various sizes, molecular masses, and elastic properties.

\section{CONCLUSION AND PERSPECTIVES}

In this work the validity of energy transfer mechanisms in the surface-influenced layer, known in water, has been evidenced as well in DPS. In dilute solutions, the kinematic conditions set by the horizontal interface keeps the same influence. However, notable differences between such shear-thinning solutions and water or Newtonian liquids are observed. Evolution of turbulence properties with polymer concentration suggest a specific behavior in the surface-influenced layer, which would need to be studied in more detail. In particular, experiments disentangling the effects of the polymer concentration and of the (low) Reynolds number should be a next step of the investigation. Starting from a critical concentration within the dilute regime, a horizontal damping mechanism arises. This damping is likely due to horizontal stresses induced by shear thinning, in a way similar to those created by surfactants. The evolution of the viscous sublayer depth depends not only on the increased viscosity or decreased $\mathrm{Re}_{T}$, but also on the shear-thinning timescale. A model can be derived, relating this evolution to the Deborah number, bulk turbulence properties, and Reynolds number in water. Additional data points are still needed in order to complete and improve this model. The knowledge brought by such measurements could later be used to measure local mass transfer mechanisms in DPS and improve the understanding of turbulent gas-liquid mass transfer in complex fluids at flat interfaces. 
TABLE II. Turbulent or grid-based Reynolds numbers computed using various reference viscosities $\left(\mu_{0}, \mu_{\infty}\right.$, solvent viscosity $\mu_{s}$, or $\mu$ at the nominal grid shear rate $\dot{\gamma} \sim f$ ). Reference viscosities used for each column are indicated as superscript. The last two columns report values of frequency (at constant stroke) and stroke (at constant frequency) that would have been necessary to achieve $\operatorname{Re}_{G}^{\mu(f)}=2025$ in polymer solutions.

\begin{tabular}{lccccccr}
\hline \hline$C_{\mathrm{XG}}[\mathrm{ppm}]$ & $\mathrm{Re}_{T}^{\mu_{\infty}}$ & $\operatorname{Re}_{T}^{\mu_{s}}$ & $\operatorname{Re}_{G}^{\mu_{0}}$ & $\operatorname{Re}_{G}^{\mu_{\infty}}$ & $\operatorname{Re}_{G}^{\mu(f)}$ & $f[\mathrm{~Hz}]$ & $S[\mathrm{~mm}]$ \\
\hline 0 & 110 & 110 & 2025 & 2025 & 2025 & 1.00 & 45 \\
10 & 138 & 137 & 1558 & 2045 & 1558 & 1.30 & 51 \\
25 & 269 & 256 & 510 & 2132 & 526 & 3.30 & 88 \\
50 & 205 & 224 & 185 & 1858 & 196 & 6.28 & 144 \\
100 & 132 & 138 & 62 & 1929 & 83 & 9.31 & 221 \\
\hline \hline
\end{tabular}

\section{APPENDIX: GRID-BASED AND TURBULENT REYNOLDS NUMBERS AT VARIOUS SCALE VISCOSITIES}

As mentioned above, $\operatorname{Re}_{T}$ values are highly dependent on the chosen reference viscosity. Values reported in Table I, for which $\mu_{0}$ was the reference viscosity, can be compared to values of $\operatorname{Re}_{T}$ based on $\mu_{\infty}$ or the solvent (water) viscosity $\mu_{s}$, denoted, respectively, by $\operatorname{Re}_{T}^{\mu_{\infty}}$ and $\operatorname{Re}_{T}^{\mu_{s}}$ (see below). As mentioned in the text, the strong decrease in $\operatorname{Re}_{T}$ is no longer observed when changing reference viscosity.

Table II shows values of the grid-based Reynolds number $\operatorname{Re}_{G}$ for all polymer concentrations, using either $\mu_{0}, \mu_{\infty}$, or the viscosity based on the grid's nominal shear rate $\mu(\dot{\gamma} \sim f)$ (reference viscosities are indicated in superscript). As a point of comparison, the grid oscillation parameters $(f, S)$ that would have been necessary to achieve match $\operatorname{Re}_{G}=2025$ with $\operatorname{Re}_{G}$ based on $\mu(f)$ are also reported (frequency variations at fixed stroke and stroke variations at fixed frequency, in the last two columns respectively). Most of the oscillation parameters are not achievable with the experimental setup used, which is limited to low frequencies and has a maximum stroke of $73 \mathrm{~mm}$. More importantly it likely would have led to polymer degradation or free surface oscillations.

[1] J. G. Janzen, H. Herlina, G. H. Jirka, H. E. Schulz, and J. S. Gulliver, Estimation of mass transfer velocity based on measured turbulence parameters, AIChE J. 56, 2005 (2010).

[2] E. A. Variano and E. A. Cowen, Turbulent transport of a high-Schmidt-number scalar near an air-water interface, J. Fluid Mech. 731, 259 (2013).

[3] T. Lacassagne, M. EL Hajem, F. Morge, S. Simoens, and J.-Y. Champagne, Study of gas liquid mass transfer in a grid stirred tank, Oil Gas Sci. Technol.-Rev. d'IFP Energies nouvelles 72, 7 (2017).

[4] J. C. R. Hunt, Turbulence structure and turbulent diffusion near gas-liquid interfaces, in Gas Transfer at Water Surfaces, edited by W. Brutsaert and G. H. Jirka, Water Science and Technology Library No. 2 (Springer, Amsterdam, 1984), pp. 67-82.

[5] B. H. Brumley and G. H. Jirka, Near-surface turbulence in a grid-stirred tank, J. Fluid Mech. 183, 235 (1987).

[6] J. Magnaudet and I. Calmet, Turbulent mass transfer through a flat shear-free surface, J. Fluid Mech. 553, 155 (2006).

[7] O. Flores, J. J. Riley, and A. R. Horner-Devine, On the dynamics of turbulence near a free surface, J. Fluid Mech. 821, 248 (2017).

[8] H. Herlina and J. G. Wissink, Isotropic-turbulence-induced mass transfer across a severely contaminated water surface, J. Fluid Mech. 797, 665 (2016).

[9] J. G. Wissink, H. Herlina, Y. Akar, and M. Uhlmann, Effect of surface contamination on interfacial mass transfer rate, J. Fluid Mech. 830, 5 (2017). 
[10] H. Yagi and F. Yoshida, Enhancement factor for oxygen absorption into fermentation broth, Biotechnol. Bioeng. 17, 1083 (1975).

[11] R. Petříček, T. Moucha, F. J. Rejl, L. Valenz, and J. Haidl, Volumetric mass transfer coefficient in the fermenter agitated by Rushton turbines of various diameters in viscous batch, Int. J. Heat Mass Transf. 115, 856 (2017).

[12] J. C. R. Hunt and J. M. R. Graham, Free-stream turbulence near plane boundaries, J. Fluid Mech. 84, 209 (1978).

[13] B. Perot and P. Moin, Shear-free turbulent boundary layers. Part 1. Physical insights into near-wall turbulence, J. Fluid Mech. 295, 199 (1995).

[14] I. Calmet and J. Magnaudet, Statistical structure of high-Reynolds-number turbulence close to the free surface of an open-channel flow, J. Fluid Mech. 474, 355 (2003).

[15] J. Bodart, J.-B. Cazalbou, and L. Joly, Direct numerical simulation of unsheared turbulence diffusing towards a free-slip or no-slip surface, J. Turbul. 11, N48 (2010).

[16] J. Magnaudet, High-Reynolds-number turbulence in a shear-free boundary layer: Revisiting the HuntGraham theory, J. Fluid Mech. 484, 167 (2003).

[17] H. Herlina and J. G. Wissink, Direct numerical simulation of turbulent scalar transport across a flat surface, J. Fluid Mech. 744, 217 (2014).

[18] H. Herlina and G. H. Jirka, Experiments on gas transfer at the air-water interface induced by oscillating grid turbulence, J. Fluid Mech. 594, 183 (2008).

[19] B. A. Toms, Some observation on the flow of linear polymer solutions through straight tubes at large reynolds numbers, in Proceedings of the First International Congress on Rheology (North Holland, Amsterdam, 1948), Vol. 2, pp. 135-141.

[20] J. L. Lumley, Drag reduction by additives, Annu. Rev. Fluid Mech. 1, 367 (1969).

[21] M. Tabor and P. G. de Gennes, A cascade theory of drag reduction, EPL (Europhys. Lett.) 2, 519 (1986).

[22] H.-D. Xi, E. Bodenschatz, and H. Xu, Elastic Energy Flux by Flexible Polymers in Fluid Turbulence, Phys. Rev. Lett. 111, 024501 (2013).

[23] M. Q. Nguyen, A. Delache, S. Simoëns, W. J. T. Bos, and M. EL Hajem, Small scale dynamics of isotropic viscoelastic turbulence, Phys. Rev. Fluids 1, 083301 (2016).

[24] A. Liberzon, M. Guala, W. Kinzelbach, and A. Tsinober, On turbulent kinetic energy production and dissipation in dilute polymer solutions, Phys. Fluids 18, 125101 (2006).

[25] G. Cocconi, E. De Angelis, B. Frohnapfel, M. Baevsky, and A. Liberzon, Small scale dynamics of a shearless turbulent/non-turbulent interface in dilute polymer solutions, Phys. Fluids 29, 075102 (2017).

[26] E. De Angelis, C. M. Casciola, R. Benzi, and R. Piva, Homogeneous isotropic turbulence in dilute polymers, J. Fluid Mech. 531, 1 (2005).

[27] A. Liberzon, On the effects of dilute polymers on driven cavity turbulent flows, Int. J. Heat Fluid Flow 32, 1129 (2011).

[28] V. K. Gupta, R. Sureshkumar, and B. Khomami, Passive scalar transport in polymer drag-reduced turbulent channel flow, AIChE J. 51, 1938 (2005).

[29] W.-H. Cai, F.-C. Li, H.-N. Zhang, X.-B. Li, B. Yu, J.-J. Wei, Y. Kawaguchi, and K. Hishida, Study on the characteristics of turbulent drag-reducing channel flow by particle image velocimetry combining with proper orthogonal decomposition analysis, Phys. Fluids 21, 115103 (2009).

[30] A. B. Rodd, D. E. Dunstan, and D. V. Boger, Characterisation of xanthan gum solutions using dynamic light scattering and rheology, Carbohyd. Polym. 42, 159 (2000).

[31] F. Garcia-Ochoa, V. E. Santos, J. A. Casas, and E. Gomez, Xanthan gum: Production, recovery, and properties, Biotech. Adv. 18, 549 (2000).

[32] B. Katzbauer, Properties and applications of xanthan gum, Polym. Degrad. Stabil. 59, 81 (1998).

[33] G. Cuvelier and B. Launay, Concentration regimes in xanthan gum solutions deduced from flow and viscoelastic properties, Carbohyd. Polym. 6, 321 (1986).

[34] N. B. Wyatt and M. W. Liberatore, Rheology and viscosity scaling of the polyelectrolyte xanthan gum, J. Appl. Polym. Sci. 114, 4076 (2009). 
[35] N. B. Wyatt, C. M. Gunther, and M. W. Liberatore, Drag reduction effectiveness of dilute and entangled xanthan in turbulent pipe flow, J. Non-Newtonian Fluid Mech. 166, 25 (2011).

[36] S. P. McKenna and W. R. McGillis, Observations of flow repeatability and secondary circulation in an oscillating grid-stirred tank, Phys. Fluids 16, 3499 (2004).

[37] M. W. McCorquodale and R. J. Munro, A method for reducing mean flow in oscillating-grid turbulence, Exp. Fluids 59, 182 (2018).

[38] T. Lacassagne, S. Simoëns, M. El Hajem, A. Lyon, and J.-Y. Champagne, Oscillating grid turbulence in shear-thinning polymer solutions, Phys. Fluids 31, 083102 (2019).

[39] S. M. Thompson and J. S. Turner, Mixing across an interface due to turbulence generated by an oscillating grid, J. Fluid Mech. 67, 349 (1975).

[40] E. J. Hopfinger and J.-A. Toly, Spatially decaying turbulence and its relation to mixing across density interfaces, J. Fluid Mech. 78, 155 (1976).

[41] A. Liberzon, M. Holzner, B. Lüthi, M. Guala, and W. Kinzelbach, On turbulent entrainment and dissipation in dilute polymer solutions, Phys. Fluids 21, 035107 (2009).

[42] Y. Wang, W.-H. Cai, T.-Z. Wei, H.-N. Zhang, L. Wang, and F.-C. Li, Proper orthogonal decomposition analysis for two-oscillating grid turbulence with viscoelastic fluids, Adv. Mech. Eng. 8, 168781401667977 (2016).

[43] L. Chiapponi, S. Longo, and M. Tonelli, Experimental study on oscillating grid turbulence and free surface fluctuation, Exp. Fluids 53, 1515 (2012).

[44] A. K. Prasad, Stereoscopic particle image velocimetry, Exp. Fluids 29, 103 (2000).

[45] T. Lacassagne, S. Simoëns, M. El Hajem, and J.-Y. Champagne, Ratiometric, single-dye, pH-sensitive inhibited laser-induced fluorescence for the characterization of mixing and mass transfer, Exp. Fluids 59, 21 (2018).

[46] B. Wieneke, PIV uncertainty quantification from correlation statistics, Meas. Sci. Technol. 26, 074002 (2015).

[47] S. P. McKenna and W. R. McGillis, The role of free-surface turbulence and surfactants in air-water gas transfer, Int. J. Heat Mass Transf. 47, 539 (2004).

[48] B.-B. Lee, E.-S. Chan, P. Ravindra, and T. A. Khan, Surface tension of viscous biopolymer solutions measured using the du Nouy ring method and the drop weight methods, Polym. Bull. 69, 471 (2012).

[49] S.-L. Young and J. A. Torres, Xanthan: effect of molecular conformation on surface tension properties, Food Hydrocolloids 3, 365 (1989).

[50] G. Hebrard, J. Zeng, and K. Loubiere, Effect of surfactants on liquid side mass transfer coefficients: A new insight, Chem. Eng. J. 148, 132 (2009).

[51] N. T. Ouellette, H. Xu, and E. Bodenschatz, Bulk turbulence in dilute polymer solutions, J. Fluid Mech. 629, 375 (2009). 